\title{
Improving Access to Sexual Health Services in General Practice Using a Hub-and-Spoke Model: A Mixed-Methods Evaluation
} \author{
Adrian Castro ${ }^{3}$, Lea Castro ${ }^{3}$, Jason $\mathrm{Wu}^{3}$, Pei Sue ${ }^{4}$, Eric Chow ${ }^{1,2}$, Marcus Chen ${ }^{1,2}$

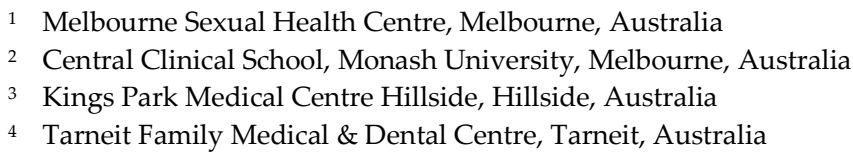

Jason J. Ong ${ }^{1,2^{*}}$, Christopher K. Fairley ${ }^{1,2}$, Ria Fortune ${ }^{1}$, Melanie Bissessor ${ }^{1}$, Chantal Maloney ${ }^{1}$, Hennie Williams ${ }^{1}$,

\begin{abstract}
Improving access to sexual health services is critical in light of rising sexually transmitted infections (STIs). We evaluated a Hub and Spoke model for improving access to sexual health services in three general practices in Victoria, Australia. The primary outcome was the impact on HIV and STI (chlamydia, gonorrhoea, syphilis) testing. Segmented linear regression analysis was conducted to examine the trends in the total HIV/STI tests before (from January 2019 to June 2020) and post-implementation (from July 2020 to July 2021). We evaluated the feasibility and acceptability of integrating this model into the general practices using semi-structured individual interviews. There was a statistically significant rise in testing for HIV and STIs in all general practices: post-implementation, there was an increase of an average of 11.2 chlamydia tests per month $(\mathrm{p}=0.026), 10.5$ gonorrhoea tests per month ( $\mathrm{p}=0.001), 4.3$ syphilis tests per month ( $\mathrm{p}=0.010$ ), and $5.6 \mathrm{HIV}$ tests per month $(p=0.010)$. Participants reported increases in knowledge level and confidence in offering STI testing and managing more variety of sexual health cases. This study demonstrated the feasibility of implementing a hub and spoke model to enable GPs to deliver sexual health care with support from a sexual health specialist service.
\end{abstract}

Keywords: HIV; sexually transmitted infection; general practice; Hub and spoke; primary care; sexual health

\section{Introduction}

The World Health Organization estimates more than a million new sexually transmitted infections (STIs) infections a day globally.[1] In Australia, there are rising rates of chlamydia, gonorrhoea and syphilis.[2] Of particular concern is the rise in congenital syphilis, which more than doubled from 7.8 per 100,000 in 2013 to 18.3 per 100,000 in 2017.[2] There are also significant social (stigma and discrimination) and economic consequences for HIV/STIs (US\$16.7 billion (range 11.8-22.1); 3.2 billion without considering HIV).[3] In recent years, there have been reports of rising antimicrobial resistance for Neisseria gonorrhoeae and Mycoplasma genitalium globally.[4]

To control HIV/STIs, healthcare systems must support earlier testing and management because a shorter duration of infectiousness has a powerful effect in reducing the incidence of HIV/STIs.[5] Most STIs are treatable, and earlier detection and management can reduce health consequences such as reproductive morbidity (e.g. infertility, abortion, preterm birth), cancer (e.g. cervical cancer from HPV), HIV transmission, and lifelong disability or death (e.g. from congenital syphilis or herpes). There is considerable evidence that HIV/STIs are driven primarily by lack of access to services rather than sexual risk behaviours. For example, in the United States, there is a lifetime HIV risk of 1 in 22 for black men and 1 in 122 for white men.[6] This large discrepancy is not primarily due to differences in condom use and partner numbers but is driven by a reduced access of black men for HIV testing, treatment, and pre-exposure prophylaxis (PrEP). Similarly, in 
Australia, though there is a reduction in HIV incidence among Australian-born gay, bisexual and other men who have sex with men (GBM), there is no change in HIV incidence among overseas-born GBM.[7] This difference may be explained by reduced access to HIV testing, treatment and PrEP for Medicare-ineligible patients, as recently arrived MSM have fewer sexual partners than Australian-born MSM.[8] Medicare is Australia's public health insurance system to provide subsidised healthcare to citizens and permanent residents. Another powerful example of the impact of access to services on infectious diseases was the widespread access to penicillin after the end of World War II in the mid1940s, with associated dramatic decreases in syphilis.[9]

General practitioners play a critical role in improving HIV/STI testing access. In Australia, it is estimated that $83 \%$ of the general population would visit a general practitioner in a year.[10] There have been several attempts at improving HIV/STI testing among GPs with mixed success.[11] The perceived lack of sexual health expertise from GPs is a barrier.[12] However, there are successes in the Australian HIV s100 prescriber system where people living with HIV receive ongoing management for HIV (including regular STI testing) within general practice.[13] Although the provision of specialised services in primary care has been adopted for other diseases using a hub-and-spoke model,[14] there have not been any published examples of providing sexual health services using a hub-and-spoke model.

The hub-and-spoke model is a network with a central facility that provides a full array of sexual health primary and specialist services ("the hub") supporting geographically dispersed secondary services ("the spokes"), which provides primary but more limited specialist services.[15] This arrangement allows for less complicated patients to be primarily served by the spokes and more complex patients to be managed at the spokes with active support or redirected to the hub when necessary. This active collaboration between the hub and its spokes could facilitate greater consistency across services in terms of efficiencies, quality of care, and enhanced accessibility of specialised services for the community. This model may be more efficient than independently replicating multiple specialist services across a geographical area and is more easily scalable (i.e., adding additional spokes when needed).

This study aimed to evaluate a Hub and Spoke model for improving access and increasing HIV/STI testing in three general practices in Victoria, Australia. The primary outcome was to evaluate the impact on HIV and STI (chlamydia, gonorrhoea, syphilis) testing. We assessed the feasibility and acceptability of integrating this model into the general practices using qualitative methods.

\section{Materials and Methods}

In 2020, Melbourne Sexual Health Centre (MSHC), a part of Alfred Health, undertook a project funded by the Victorian Department of Health and Human Services in response to the Victorian Review of Sexual Health Services, which recommended the development of HIV/STI services provided in a decentralised hub and spoke model where Melbourne Sexual Health Centre served as the specialist hub for HIV/STI testing and treatment supporting GP spokes that provide primary care HIV/STI testing and treatment. The training phase of the project took place between May 2020 and July 2020. The support Melbourne Sexual Health Centre delivered to these three GP partner services is provided in Appendix 1. Three suburban GP practices were identified and included in the study located West of Melbourne (Clinic 1), South of Melbourne (Clinic 2) and North-West of Melbourne (Clinic 3). These locations were selected based on syphilis prevalence data that syphilis infection has spread to outer metropolitan areas of Melbourne.[16]

We extracted data from the pathology provider on the number of tests (HIV, syphilis, chlamydia, gonorrhoea) and test positivity for each clinic from two time periods: 1) Preimplementation phase: January 2019 to June 2020; and 2) Implementation phase: July 2020-February 2021. Descriptive statistics were used to analyse the data. We used Student's t-test to compare the average number of tests for each pathogen pre- 
implementation and implementation phase. Segmented linear regression analysis was conducted to examine the trends in the total HIV/STI tests before (from January 2019 to June 2020) and post-implementation (from July 2020 to July 2021).

A total of 33 semi-structured individual interviews were conducted from May to June 2020, before the training: 5 from Clinic 1, 13 from Clinic 2, and 15 from Clinic 3. Interviews explored the staff's current knowledge, attitude, and practice related to sexual health; current challenges in providing sexual health care in the general practice; and training needs. A second interview was conducted among staff who had experience providing the sexual health services in the respective clinics in August and September 2020. Interviews explored their experiences in delivering sexual health care as part of the Hub and Spoke model; feedback on the training received for the implementation of the sexual health service; changes in their knowledge, attitude and practice as a result of participating in the training; and ongoing support needs. All interviews were recorded and data analysed using a content analysis approach to summarise the main themes. Themes were generated from the data without a pre-existing framework. No financial reimbursement was offered to interviewees.

As this was part of a quality improvement and evaluation activity, we received a waiver from the Alfred Hospital Human Research Ethics Committee. Funding was provided from the Victorian Department of Health and Human Services. The researchers are independent of the funders who did not have any role in the design, conduct, analysis or decision to publish the study. The lead author (JO) had full access to all the data. All relevant data are presented in this manuscript. Further details can be obtained by contacting the corresponding author.

\section{Results}

Table 1 summarises the average number of HIV/STI tests per GP clinic, pre-and posttraining. It demonstrates a significant increase in testing for chlamydia, gonorrhoea, syphilis and HIV across all three general practices. Pre-implementation, there was no significant change in the total chlamydia tests ( $\mathrm{p}=0.246$, Figure 1 ). Post-implementation, there was an immediate increase in the number of chlamydia tests of 33.7 in the first month after the GP hub and spoke was launched. This was followed by a significant increase of an average of 11.2 tests per month $(\mathrm{p}=0.026)$.

Table 1. The average number of HIV/STI tests. Note: SD = standard deviation

\begin{tabular}{|c|c|c|c|}
\hline & $\begin{array}{c}\text { Before implementation - Aver } \\
\text { number per month (SD) }\end{array}$ & $\begin{array}{l}\text { ter implementation - Average } \\
\text { number per month (SD) }\end{array}$ & p value \\
\hline \multicolumn{4}{|l|}{ Chlamydia } \\
\hline Clinic 1 & $16.7(5.6)$ & 35.5 (15.5) & $<0.0001$ \\
\hline Clinic 2 & $13.1(4.3)$ & $55.5(14.8)$ & $<0.0001$ \\
\hline Clinic 3 & $31.8(6.7)$ & $64.1(37.4)$ & $<0.0001$ \\
\hline Total & $61.6(12.5)$ & $155.1(57.6)$ & $<0.0001$ \\
\hline \multicolumn{4}{|c|}{ Gonorrhoea } \\
\hline Clinic 1 & $16.0(5.6)$ & $34.9(13.8)$ & $<0.0001$ \\
\hline Clinic 2 & $11.8(3.8)$ & $51.1(12.5)$ & $<0.0001$ \\
\hline Clinic 3 & $24.3(5.8)$ & $63.3(38.1)$ & $<0.0001$ \\
\hline Total & $52.2(11.3)$ & $114.5(33.0)$ & $<0.0001$ \\
\hline \multicolumn{4}{|l|}{ Syphilis } \\
\hline Clinic 1 & $20.0(5.2)$ & $24.3(7.9)$ & $<0.0001$ \\
\hline Clinic 2 & $12.7(5.9)$ & $33.7(7.4)$ & $<0.0001$ \\
\hline Clinic 3 & $20.5(7.1)$ & $35.5(16.1)$ & $<0.0001$ \\
\hline Total & $53.2(12.4)$ & $93.5(24.6)$ & $<0.0001$ \\
\hline \multicolumn{4}{|l|}{ HIV } \\
\hline Clinic 1 & $21.1(5.0)$ & $24.6(7.9)$ & $<0.0001$ \\
\hline Clinic 2 & $23.3(6.6)$ & $34.3(7.3)$ & $<0.0001$ \\
\hline Clinic 3 & $24.4(11.6)$ & $40.4(18.1)$ & $<0.0001$ \\
\hline Total & $68.8(17.6)$ & $99.3(26.5)$ & $<0.0001$ \\
\hline
\end{tabular}




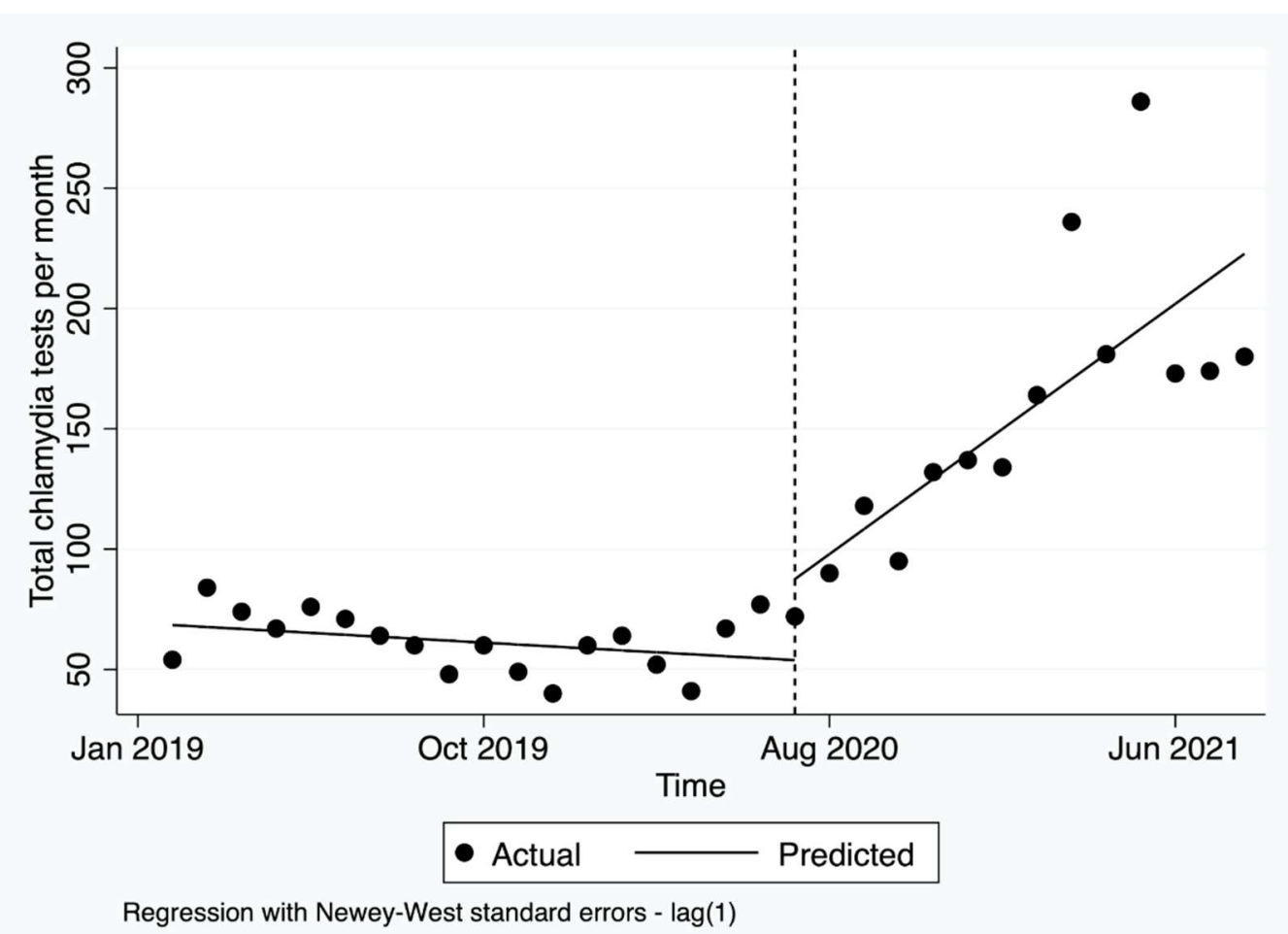

Figure 1. Number of tests for chlamydia before and after the Hub and Spoke model was launched.

Pre-implementation, there was no significant change in the total gonorrhoea tests $(\mathrm{p}=0.826$, Figure 2). Post-implementation, there was an immediate increase in the number of gonorrhoea tests of 38.4 in the first month after the GP hub and spoke was launched. This was followed by a significant increase of an average of 10.5 tests per month $(\mathrm{p}=0.001)$.

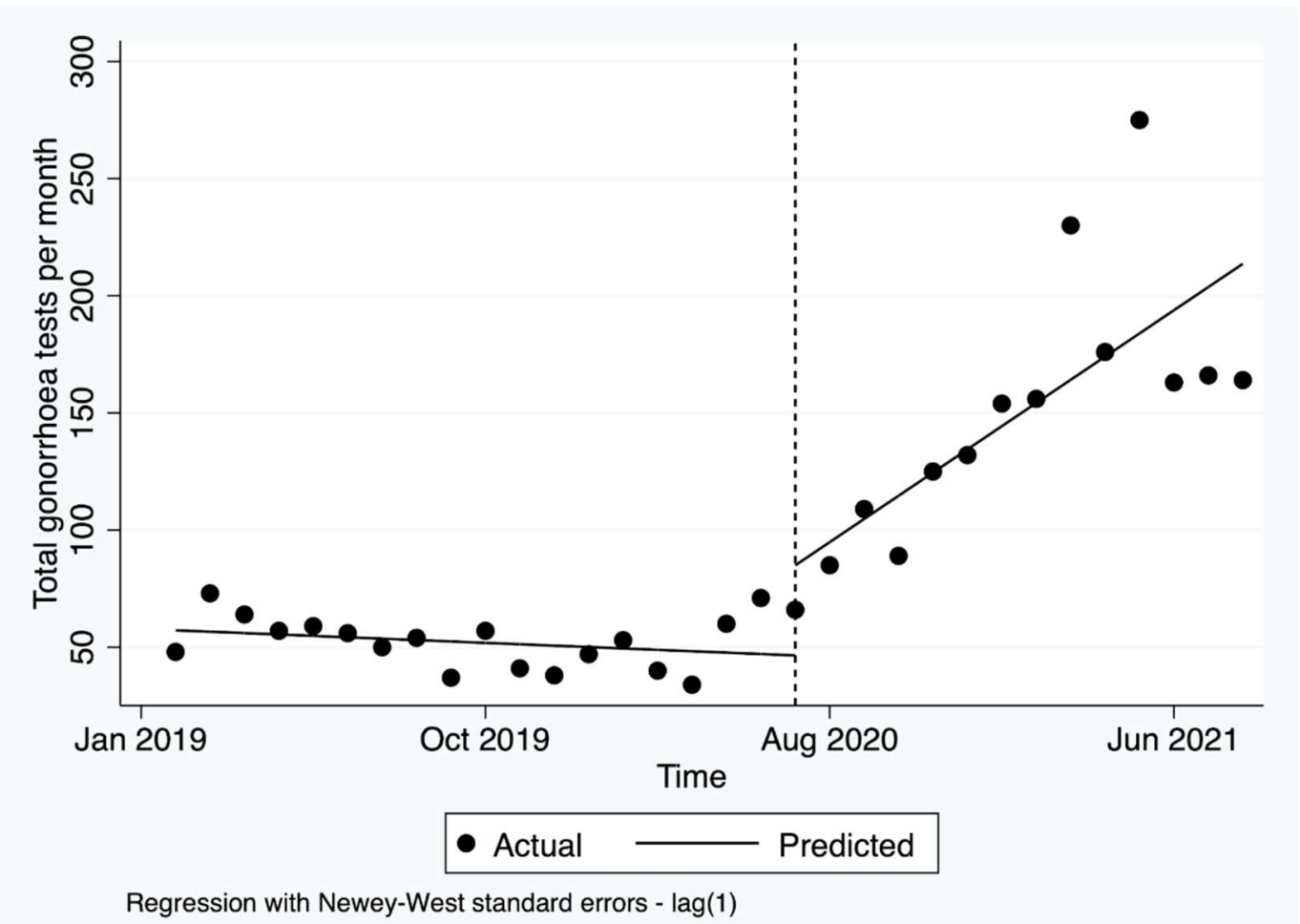

Figure 2. Number of tests for gonorrhoea before and after the Hub and Spoke model was launched. 
Pre-implementation, there was no significant change in the total syphilis tests ( $p=0.122$, Figure 3). Post-implementation, there was an immediate increase in the number of syphilis tests of 27.0 in the first month after the GP hub and spoke was launched. This was followed by a significant increase of an average of 4.3 tests per month $(\mathrm{p}=0.010)$.

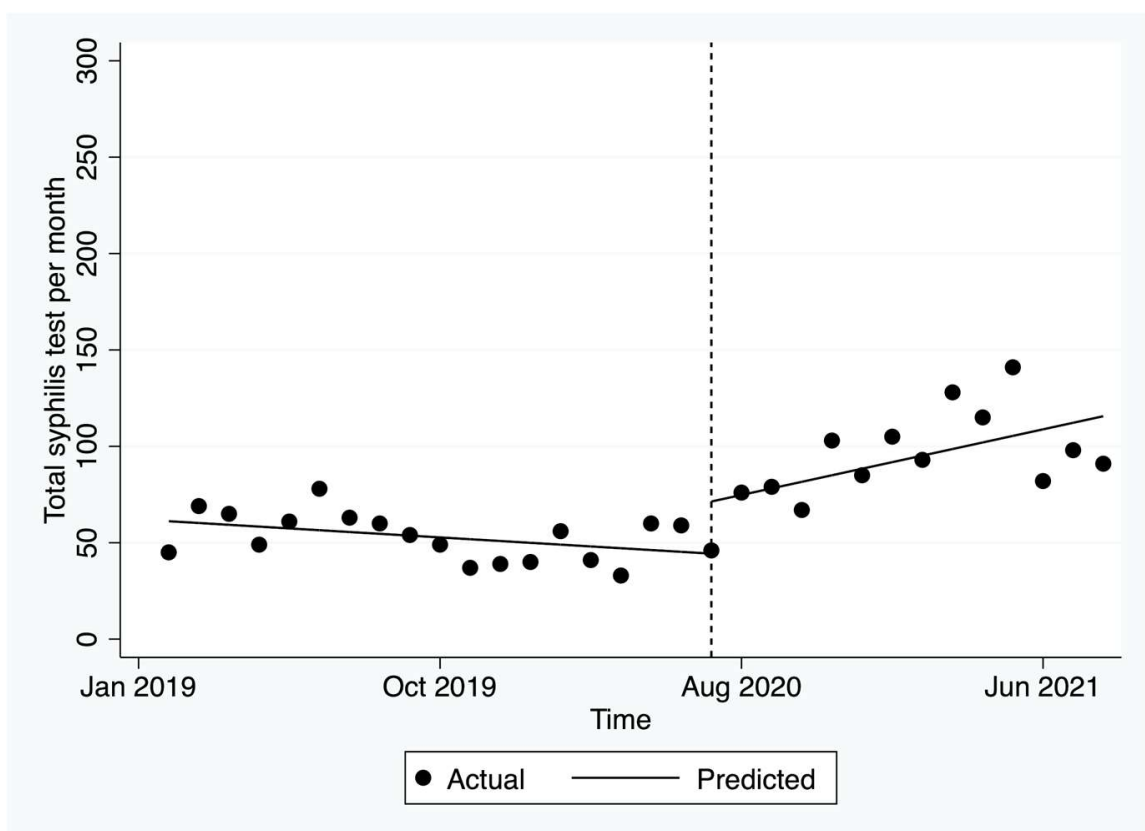

Figure 3. Number of tests for syphilis before and after the Hub and Spoke model was launched.

Pre-implementation, there was a significant decrease of HIV tests of 2.3 per month $(p=0.002$, Figure 4). Post-implementation, there was an immediate increase in HIV tests of 30.4 in the first month after the GP hub and spoke was launched. This was followed by a significant increase of an average of 5.6 tests per month $(p=0.010)$.

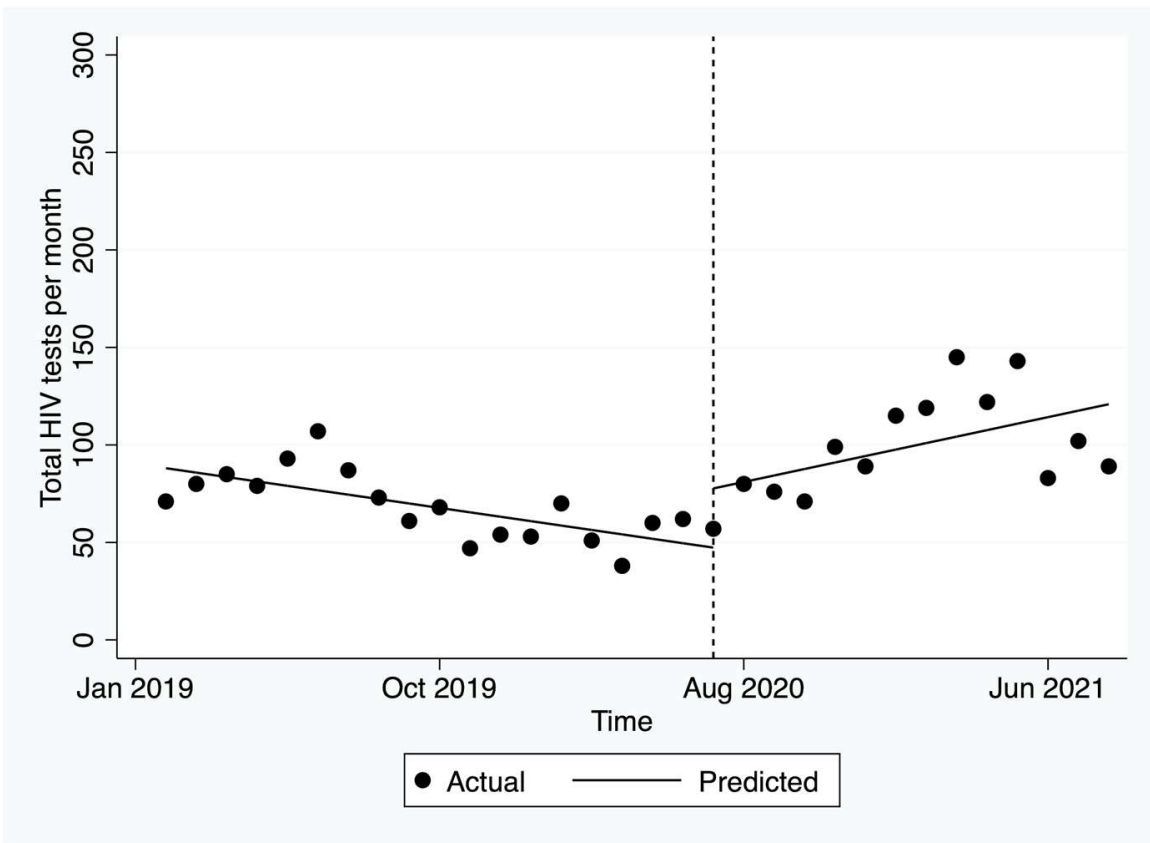

Figure 4. Number of tests for HIV before and after the Hub and Spoke model.

There was no significant change in test positivity for chlamydia, gonorrhoea, syphilis and HIV (Appendix 2). Results from the qualitative interviews are presented in Appendix 
3. Participants described their training needs, other challenges associated with delivering sexual health services, and areas for improvement for future Hub and Spoke models.

\section{Discussion}

We evaluated a program that implemented a hub and spoke method to deliver sexual health services in Victoria, Australia. We found that the hub and spoke model led to an early and sustained increase in chlamydia, gonorrhoea, syphilis and HIV testing. This is despite the social restrictions imposed in Victoria because of the COVID-pandemic. Our study adds to the scant literature on how a hub and spoke model can be used to improve access to sexual health services.[17]

A key target of the Fourth Australian National Sexually Transmissible Infections Strategy (2018-2022) is to increase STI testing coverage.[18] Enabling timely access to testing and treatment requires new strategies and policies to enable accessible STI care. There are several ways to configure HIV/STI testing and management services, including the potential for integrating novel service delivery models such as virtual STI clinics,[19] postal service,[20] or pharmacy-based services.[21] However, decision-makers have limited evidence on which programs are likely to be used by those at greatest need, which are most cost-effective, and which should be scaled up. Our findings demonstrate that the GP Hub and Spoke model significantly increased and sustained testing (at least for one year) for chlamydia, gonorrhoea, syphilis and HIV. This is despite the restrictions related to the COVID-19 pandemic in Victoria.

There are several advantages to the GP Hub and Spoke model. Strengthening general practices to provide sexual health care can help destigmatise sexual health services by providing them through GPs who are already accessible and trusted by the community. Patients who book in for a sexual health consultation with their GP may feel more comfortable discussing sexual health matters, knowing their GP is interested and skilled in providing this care.[22] Normalisation and destigmatisation of STI testing are critical for reducing the burden of STIs and promoting sexual health. The hub and spoke model could also be a more efficient use of specialist services, i.e. complex cases from spokes can be redirected to the hub and vice versa - send "basic" cases to the spokes. Given that most of the population encounter a GP every year (83.2\% in 2019/2020),[23] there is an opportunity for opportunistic screening when people present with non-sexual health-related complaints or issues - reaching people who may not attend public sexual health services. In addition, GPs can offer additional services such as vaccination, prevention (PrEP), counselling, mental health, substance use supports for those who need these services. Scaling up the hub and spoke model, particularly to remote and rural areas can increase the accessibility of sexual health services to these underserved populations.

Our model of care uses the nurse to do the initial assessment. This is beneficial for embedding STI testing in the practice, creating extra capacity for patient appointments, and workforce development for sexual health nurses.[24] Our study also identified several challenges in creating a hub and spoke model for sexual health services. First, it is not clear what are the predictors or markers of a successful spoke are. In our experience thus far, we found three elements were important: 1) Commitment and involvement of the principal GP; 2) One or more staff have a particular interest in delivering sexual health care to their patients; and 3) The governance structure of the practice/service means that practice staff are consulted, involved and supported to participate in delivering sexual health care. This is critical, as the training time and resource investment are significant (Appendix 1). Second, building trust and relationships with the local communities to create a safe space for sexual health services will take time. This includes appropriate and targeted marketing. Unlike traditional hub-and-spoke organisational designs,[14] we allowed spokes to have relative independence in adapting the sexual health service to the GPs' needs. This may lead to inconsistency across operations but functionally more suitable to local needs. Our study was not a hierarchical structure with authority of hubs over the spokes. This means that patients may not have similar experiences across all services 
(not like a franchise). Therefore, there may be some risks related to unmet expectations of patients expecting the same level of service/expertise when attending a specialist service, with the potential to tarnish the reputation of the organisations if a high-quality service is not sustained. Mitigation of this risk requires ongoing quality assurance and support. Third, there may be direct impacts on the patient flow in the GP practice. For example, patients would initially see the nurse (task-shifting) upskilled to provide sexual health care. Fourth, if there is a high staff turnover in the GP clinic, this necessitates frequent and ongoing training for new staff.

Our results must be read in light of some limitations. The evaluation was conducted during major disruptions to primary care services because of COVID-19 related social distancing restrictions. General practices during this period had to prioritise COVID-related activities (including delivering vaccines) and transition from in-person to telehealth services. Nevertheless, we still observed a significant rise in HIV/testing post-implementation. However, we did not observe any impact on test positivity which could either reflect a lower incidence of STIs because of declines in sexual activity[25] or the increase in testing was among low-risk populations. There will be ongoing monitoring of HIV/STI testing and positivity rates as the hub and spoke model is scaled up in Victoria.

\section{Conclusions}

This study demonstrated that GPs are well placed to deliver sexual health care with specialist services providing support and training through a Hub and Spoke Model. This ongoing and synergistic collaboration has led to a sustained increase in HIV and STI testing.

Supplementary Materials: Appendix 1: Features of the training for the spokes; Appendix 2: Test positivity for chlamydia, gonorrhea, syphilis and HIV; Appendix 3: Results from qualitative interviews

Author Contributions: Conceptualization, J.O., C.F., R.F., M.C.; methodology, J.O., M.C.; formal analysis, J.O.; investigation, J.O.; writing - original draft preparation, J.O.; writing-J.O., C.F., R.F., M.B., C.M., H.W., A.C., L.C., J.W., P.S., E.C., M.C.; supervision, M.C.; project administration, R.F., M.B., C.M., H.W.; funding acquisition, M.C., R.F., C.F. All authors have read and agreed to the published version of the manuscript.

Funding: This research was funded by the Victorian Department of Health and Human Services.

Institutional Review Board Statement: The study was conducted in accordance with the Declaration of Helsinki. Ethical review and approval were waived for this study due to this study being a part of a quality improvement and evaluation activity.

Informed Consent Statement: Patient consent was waived due to non-identifiable data received from the pathology company.

Data Availability Statement: All relevant data are presented in this manuscript. Further details can be obtained by contacting the corresponding author.

Acknowledgments: We thank Darren McKee from the Australian Clinical Labs for providing the data on HIV/STI testing from the general practices.

Conflicts of Interest: The authors declare no conflict of interest. The funders had no role in the design of the study; in the collection, analyses, or interpretation of data; in the writing of the manuscript, or in the decision to publish the results.

\section{References}

1. Rowley, J.; Vander Hoorn, S.; Korenromp, E. L.; Low, N.; Unemo, M.; Abu-Raddad, L. J.; Chico, R. M.; Smolak, A.; Newman, L.; Gottlieb, S.; Thwin, S.; Broutet, N.; Taylor, M., Chlamydia, gonorrhoea, trichomoniasis and syphilis: global prevalence and incidence estimates, 2016. Bull World Health Organ 2019. 
2. University of New South Wales. HIV, viral hepatitis and sexually transmitted infections in Australia: Annual Surveillance Report 2018. https://kirby.unsw.edu.au/report-type/annual-surveillance-reports (18th Jan 2019),

3. Chesson, H. W.; Mayaud, P.; Aral, S. O., Sexually Transmitted Infections: Impact and Cost-Effectiveness of Prevention. In Major Infectious Diseases, rd; Holmes, K. K.; Bertozzi, S.; Bloom, B. R.; Jha, P., Eds. Washington (DC), 2017.

4. Unemo, M.; Jensen, J. S., Antimicrobial-resistant sexually transmitted infections: gonorrhoea and Mycoplasma genitalium. Nat Rev Urol 2017, 14, (3), 139-152.

5. Australian government department of health. Fourth National Sexually Transmissible Infections Strategy $2018-2022$. https://www1.health.gov.au/internet/main/publishing.nsf/Content/ohp-bbvs-1/\$File/STI-Fourth-Nat-Strategy-2018-22.pdf (25th March 2020),

6. Hess, K. L.; Hu, X.; Lansky, A.; Mermin, J.; Hall, H. I., Lifetime risk of a diagnosis of HIV infection in the United States. Ann Epidemiol 2017, 27, (4), 238-243.

7. New South Wales Government HIV Surveillance Data Reports. Second Quarter Data Report 2019. https://www.health.nsw.gov.au/endinghiv/Pages/tools-and-data.aspx (1 Nov 2019),

8. Blackshaw, L. C. D.; Chow, E. P. F.; Varma, R.; Healey, L.; Templeton, D. J.; Basu, A.; Turner, D.; Medland, N. A.; Rix, S.; Fairley, C. K.; Chen, M. Y., Characteristics of recently arrived Asian men who have sex with men diagnosed with HIV through sexual health services in Melbourne and Sydney. Aust N Z J Public Health 2019, 43, (5), 424-428.

9. Jasek, E.; Chow, E. P.; Ong, J. J.; Bradshaw, C. S.; Chen, M. Y.; Hocking, J. S.; Lee, D.; Phillips, T.; Phillips, T.; Temple-Smith, M.; Fehler, G.; Fairley, C. K., Sexually Transmitted Infections in Melbourne, Australia from 1918 to 2016: nearly a century of data. Commun Dis Intell Q Rep 2017, 41, (3), E212-E222.

10. Australian Bureau of Statistics. Patient experiences in Australia: Summary of Findings, $2019-20$ financial year. https://www.abs.gov.au/statistics/health/health-services/patient-experiences-australia-summary-findings (5th Jan 2020),

11. Hocking, J. S.; Temple-Smith, M.; Guy, R.; Donovan, B.; Braat, S.; Law, M.; Gunn, J.; Regan, D.; Vaisey, A.; Bulfone, L.; Kaldor, J.; Fairley, C. K.; Low, N.; Consortium, A. C., Population effectiveness of opportunistic chlamydia testing in primary care in Australia: a cluster-randomised controlled trial. Lancet 2018, 392, (10156), 1413-1422.

12. Llewellyn, C.; Pollard, A.; Miners, A.; Richardson, D.; Fisher, M.; Cairns, J.; Smith, H., Understanding patient choices for attending sexually transmitted infection testing services: a qualitative study. Sex Transm Infect 2012, 88, (7), 504-9.

13. Smith, D. E.; Woolley, I. J.; Russell, D. B.; Bisshop, F.; Furner, V., HIV in practice: current approaches and challenges in the diagnosis, treatment and management of HIV infection in Australia. HIV Med 2018, 19 Suppl 3, 5-23.

14. Devarakonda, S., Hub and spoke model: making rural healthcare in India affordable, available and accessible. Rural Remote Health 2016, 16, (1), 3476.

15. Elrod, J. K.; Fortenberry, J. L., Jr., The hub-and-spoke organization design: an avenue for serving patients well. BMC Health Serv Res 2017, 17, (Suppl 1), 457.

16. Aung, E. T.; Chen, M. Y.; Fairley, C. K.; Higgins, N.; Williamson, D. A.; Tomnay, J. E.; Cook, K. A.; Peel, J.; Dharmakulasinghe, V.; Alpren, C.; Chow, E. P. F., Spatial and Temporal Epidemiology of Infectious Syphilis in Victoria, Australia, 2015-2018. Sex Transm Dis 2021, 48, (12), e178-e182.

17. Desai, M.; Littler, J. E.; Samuel, M.; Baker, D. P.; Loader, P. B.; Singh, S. P.; Bradbeer, C. S., How to set up a remotely supported hub and spoke sexual health service for a military population. Sex Transm Infect 2015, 91, (8), 545-7.

18. Australian government department of health. Fourth National Sexually Transmissible Infections Strategy. . https://www1.health.gov.au/internet/main/publishing.nsf/Content/ohp-bbvs-1/\%24File/STI-Fourth-Nat-Strategy-201822.pdf (27th October 2021), 
19. Wilson, E.; Free, C.; Morris, T. P.; Syred, J.; Ahamed, I.; Menon-Johansson, A. S.; Palmer, M. J.; Barnard, S.; Rezel, E.; Baraitser, P., Internet-accessed sexually transmitted infection (e-STI) testing and results service: A randomised, single-blind, controlled trial. PLoS Med 2017, 14, (12), e1002479.

20. Victorian Department of Health and Human Services. Victorian Sexual Health and Service Needs. . https://www2.health.vic.gov.au/about/publications/researchandreports/review-vic-sexual-health-service-needs-oct-2019 (31st March 2020),

21. Anderson, C.; Thornley, T., A pharmacy-based private chlamydia screening programme: results from the first 2 years of screening and treatment. Int J Clin Pharm 2011, 33, (1), 88-91.

22. Baker, J. R.; Arnold-Reed, D. E.; Brett, T.; Hince, D. A.; O'Ferrall, I.; Bulsara, M. K., Perceptions of barriers to discussing and testing for sexually transmitted infections in a convenience sample of general practice patients. Aust J Prim Health 2013, 19, (2), 98-101.

23. Australian Bureau of Statistics. Patient experiences in Australia: summary of findings. https://www.abs.gov.au/statistics/health/health-services/patient-experiences-australia-summary-findings/latest-release (14th September 2021),

24. Lorch, R.; Hocking, J.; Guy, R.; Vaisey, A.; Wood, A.; Lewis, D.; Temple-Smith, M.; Consortium, A. C., Practice nurse chlamydia testing in Australian general practice: a qualitative study of benefits, barriers and facilitators. BMC Fam Pract $2015,16,36$.

25. Coombe, J.; Kong, F. Y. S.; Bittleston, H.; Williams, H.; Tomnay, J.; Vaisey, A.; Malta, S.; Goller, J. L.; Temple-Smith, M.; Bourchier, L.; Lau, A.; Chow, E. P. F.; Hocking, J. S., Love during lockdown: findings from an online survey examining the impact of COVID-19 on the sexual health of people living in Australia. Sex Transm Infect 2021, 97, (5), 357-362. 